\title{
Effect of Flavonoid Rich Root Extract Of Glycyrrhiza glabra on Gastric Emptying and Gastrointestinal Transit in Albino Wistar Rats
}

\author{
Sasikumar Murugan ${ }^{1}$, Chandrasekaran Chinampudur Velusami ${ }^{2}$, Bharathi Bethapudi ${ }^{3}$, Ramanaiah Illuri ${ }^{1}$ and Deepak \\ Mundkinajeddu ${ }^{2}$ \\ ${ }^{1} J u n i o r$ Research officer, R\&D Biology,Natural Remedies Private Limited, Karnataka, India \\ ${ }^{2} \mathrm{Head}$ of R\&D Biology, Natural Remedies Private Limited, Karnataka, India \\ ${ }^{3}$ Senior Research Officer, R\&D Biology, Natural Remedies Private Limited, Karnataka, India
}

Received: February 13, 2017; Accepted:April 5, 2017; Published: April 24, 2017

*Corresponding author: Chandrasekaran Chinampudur Velusami, Head of Biology, R\&D center, Natural Remedies Private Limited, 5B, Veerasandra Indl. Area, 19th K. M. Stone, Hosur road, Electronic City Post, Bangalore, 560100, Karnataka, India, Tel: +91-9945887240; Fax: +91 - 80- 40209817; E-mail: cvc@ naturalremedy.com

\begin{abstract}
Background: Glycyrrhiza glabra is often used in the management of gastrointestinal disorders. The mechanism for its beneficial actions in gastrointestinal system remains to be elucidated.

Objective: To evaluate the gastroprokinetic effect of flavonoid rich root exrtact of Glycyrrhiza glabra (GutGard) and glabridin in albino Wistar rats using phenol red meal model.

Materials and Methods: Rats were orally treated with Gutgard or glabridin for 8 consecutive days. On $8^{\text {th }}$ day, rats were administered phenol red meal $2 \mathrm{~h}$ post GutGard or glabridin administration. Percentage gastric emptying and gastrointestinal transit were measured immediately and 20 minutes after phenol red meal administration.

Results: Rats treated with GutGard exhibited statistically significant increase in percentage gastric emptying as well as gastrointestinal transit over the normal control group. Gastric emptying of GutGard and glabridin is comparable to domperidone, a potent prokinetic agent.

Conclusion: The study findings revealed that, GutGard can alleviate gastrointestinal symptoms of fuctional dyspepsia via prokinetic activity as evident from increase of gastric emptying and gastrointestinal transit. This prokinetic effects of Glycyrrhiza glabra might be contributed by galbridin.
\end{abstract}

Key words: Prokinetic activity; Fuctional dyspepsia; GutGard; Phenol red; Glycyrhizza glabra

\section{Introduction}

Functional dyspepsia (FD) is a disorder characterized by upper abdominal pain or discomfort in the absence of organic disease but often linked to a motility disorder, occurs very commonly in the general population. Delayed gastric emptying is considered as a major pathophysiologic mechanism in FD, and it has been identified in $29 \%-59 \%$ of patients with FD [1]. Although several agents have been evaluated for FD, drugs approved by FDA are not available for treatment of FD. Agents that improve motility (prokinetic agents) have been recommended as empirical treatment [2].

Prokinetic agents such as antidopaminergics (eg: metoclopramide, domperidone, levosulpiride) and serotonin 5-HT4 receptor agonists (eg: tegaserod) have shown to have modest effects in the treatment of FD. However they have their own disadvantages due to limited clinical effects and unwanted side effects like prolonged QT intervals etc. This has led to focus on phytotherapy that could improve gastric emptying thereby ameliorate FD symptoms $[2,3]$.

GutGard, a flavonoid rich root extract of Glycyrrhiza glabra has been developed and proved to be beneficial for the management in ameliorating the symptoms of FD. However the mechanisms behind the effectiveness of GutGard for the management of FD were not explored. This study is to test the hypothesis that, GutGard would have ameliorated FD symptoms via improving gastric emptying and gastrointestinal transit. The present study was conducted to evaluate the gastroprokinetic effect of flavonoid rich root exrtact of Glycyrrhiza glabra (GutGard) in rats using phenol red meal model. Further, the effect of glabridin on gastric emptying and gastrointestinal transit were also explored.

\section{Material And Methods}

\section{Drugs and chemicals}

Domperidone (Cipla Ltd., India), phenol red sodium (Loba Chemie Pvt. Ltd., India.) were used and all the other chemicals used in the experiment like carboxy methyl cellulose monosodium salt (CMC), sodium hydroxide and trichloroacetic acid were purchased from Hi-Media Laboratories Pvt. Ltd., India.

\section{Test Substance}

GutGard is a flavonoid rich, root extract of Glycyrrhiza glabra developed by Natural Remedies, Bangalore, India. GutGard has following phytochemical specifications, namely, 
glabridin $(\geq 3.5 \% \mathrm{w} / \mathrm{w})$, glabrol $(\geq 0.5 \% \mathrm{w} / \mathrm{w})$, eicosanyl caffeate $(\geq 0.1 \% \mathrm{w} / \mathrm{w})$, docosylcaffeate $(\geq 0.1 \% \mathrm{w} / \mathrm{w})$, glycyrrhizin $(\leq 0.5 \% \mathrm{w} / \mathrm{w})$, and total flavonoids $(\geq 10 \% \mathrm{w} / \mathrm{w})$ [5].

\section{Animals}

Male albino Wistar rats (190-200 g) bred at R\&D Centre, Natural Remedies Pvt Ltd, was acclimatized for 7 days prior to experimentation. The animals during experimentation were housed under standard husbandry conditions of temperature $\left(22 \pm 3^{\circ} \mathrm{C}\right)$, relative humidity $(30 \%-70 \%)$ and light: dark cycle of 12:12 h. Rats were allowed free access to pellet feed and UV purified water. All the experimental protocols (Protocol approval number: IAEC/PCL/06/06.15) were approved by the Institutional Animal Ethics Committee (IAEC) of Natural Remedies Private Limited, Bangalore, and conducted according to the Committee for the Purpose of Control and Supervision of Experiments on Animals (CPCSEA) guidelines.

\section{Experimental procedure}

\section{Preparation of Phenol Red Test Meal}

Carboxy methyl cellulose was dissolved in water at about $80^{\circ} \mathrm{C}$ and prepared to a final concentration of $1.5 \%$. The solution was stirred until dissolved and phenol red $(50 \mathrm{mg} / 100 \mathrm{ml})$ was added to the stirring solution. The mixture was then brought down to $37^{\circ} \mathrm{C}$.

\section{Study Design}

Male albino Wistar rats were randomly allotted to eight groups each consisting of six animals. Group I was adminstered with CMC and Group II was administered Domperidone (10 $\mathrm{mg} / \mathrm{kg}$ ) as a single dose on day 8, while Group III, IV, V were adminstered various dose levels of GutGard (6.25, 12.5 and 25 $\mathrm{mg} / \mathrm{kg}$ ) respectively and Group VI, VII, VIII were adminstered various dose levels of Glabridin (2.5, 5 and $10 \mathrm{mg} / \mathrm{kg}$ ) respectively for 8 consecutive days. On day 8 , sixteen hours fasted rats were administered phenol red meal ( $2 \mathrm{ml} /$ animal $) 2 \mathrm{~h}$ post the test substance or reference drug administration. Immediately and 20 minutes after phenol red meal administration, rats from each group were sacrificed by cervical dislocation under anesthesia. Abdomen was opened, gastroesophageal junction and the pylorus ends were clamped, then stomach was dissected out and placed in $100 \mathrm{ml}$ of $0.1 \mathrm{~N} \mathrm{NaOH}$ and homogenised. The homogenate is kept at room temperature for an hour and $5 \mathrm{ml}$ of the homogenate, was added to $0.5 \mathrm{ml}$ of Trichloroacetic acid $(20 \% \mathrm{w} / \mathrm{v}$ ) and centrifuged at $3000 \mathrm{rpm}$ for $20 \mathrm{mins}$. To $1 \mathrm{ml}$ of the supernatant $4 \mathrm{ml}$ of the $0.5 \mathrm{~N} \mathrm{NaOH}$ was added and the absorbance of the resultant pink colour liquid was measured using Versamax (molecular devices) microplate reader at $560 \mathrm{~nm}$ wavelength. The percentage of gastric emptying in 20 minutes was calculated by the formula:

Percentage Gastric Emptying (\%) = (1- X/Y) multiplied by 100

$\mathrm{X}$ : absorbance of phenol red recovered from stomach of rats sacrificed 20 minutes after test meal

Y: absorbance of phenol red recovered from stomach of rats sacrificed at $0^{\text {th }}$ minute (immediately) after test meal

\section{Gastrointestinal Transit (GIT)}

Intestine was dissected out from duodenum to ileoceacal junction and the distance travelled by phenol red and total length of the intestine (from duodenum to ileo-ceacal junction) was recorded. The gastrointestinal transit (GIT) was calculated by the formula:

GIT $(\%)=($ Distance travelled by phenol red meal in cms / Total length of the small intestine in cms) multiplied by 100 .

\section{Statistical Analysis}

The data were processed using statistical software IBM SPSS version 20. Percentage gastric emptying and gastrointestinal transit were expressed as mean \pm SEM and analyzed statistically using One Way ANOVA followed by post-hoc Bonferroni test. P $\leq$ 0.05 was considered to be statistically significant.

\section{Results}

\section{Percentage Gastric Emptying (\%)}

Twenty minutes after the administration of phenol red meal to the rats, the percentage gastric emptying in control group was found to be $53.33 \%$ and group administered with single dose of domperidone (10 mg/kg p.o.) was found to be $87.03 \%$. Groups administered with GutGard and glabridin at all dose levels exhibited statistically significant increase of gastric emptying over control group (Table 1).

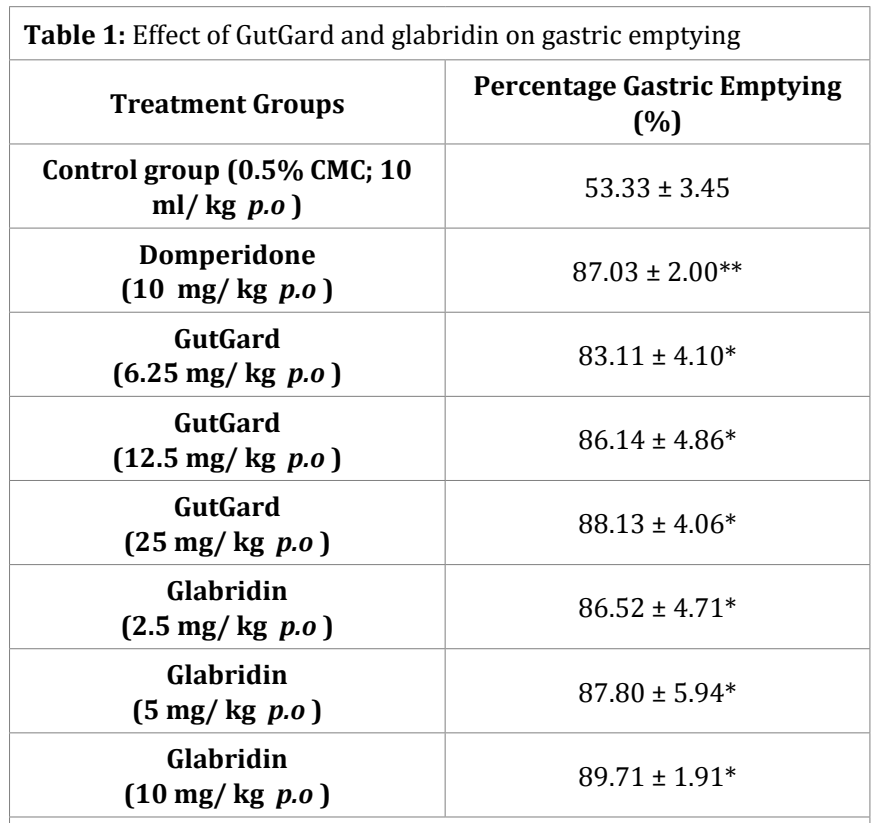

Effect of GutGard and glabridin on Percentage gastric emptying in rats administered with phenol red test meal. Data were expressed as mean $\pm \mathrm{SEM}$ (Group I - V; n=12 (pooled data from two independent experiments) and Group VI-VIII; $n=6$ ). ${ }^{*} \mathrm{p}<0.05$ significantly different from normal control group; ${ }^{* *} \mathrm{p}<$ 0.01 significantly different from normal control group. 


\section{Gastrointestinal transit (\%)}

Groups administered with domperidone and GutGard at all dose levels demonstrated statistically significant increase in gastrointestinal transit (\%) compared to the control group. Glabridin treated groups showed non-significant increase in gastrointestinal transit (\%) compared to the control group. Gastrointestinal transit (GIT) of control group was found to be $62.62 \%$ and group administered with single dose of domperidone (10 mg/kg p.o.) was found to be $88.24 \%$. GutGard treated groups $(6.25,12.5$ and $25 \mathrm{mg} / \mathrm{kg}$ p.o.) showed significant increase $(77.76 \%, 79.38 \%$ and $83.60 \%)$ of gastrointestinal transit compared to the control group. Groups treated with Glabridin (2.5, 5 and $10 \mathrm{mg} / \mathrm{kg}$ p.o.) exhibited non-significant increase $(71.25 \%, 72.83 \%$ and $73.87 \%)$ of gastrointestinal transit compared to control group (Figure 1).

Figure 1: Effect of GutGard and glabridin on gastrointestinal transit

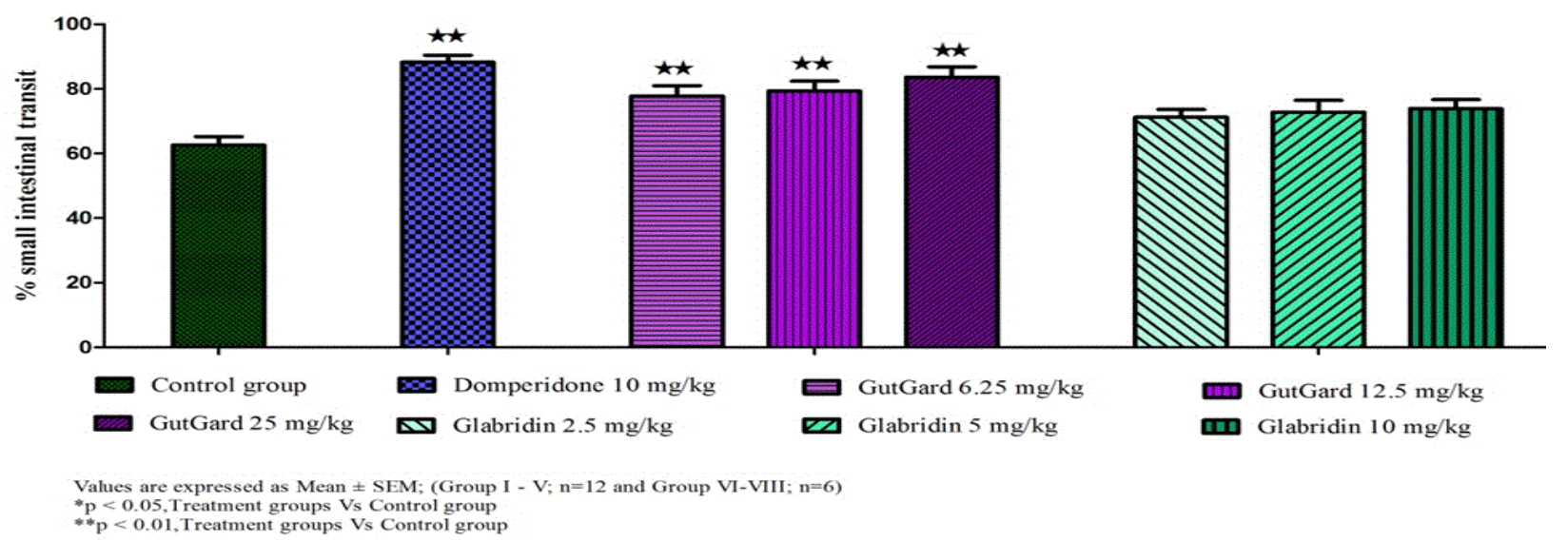

Figure 1: Effect of GutGard and glabridin on percentage gastrointestinal transit in rats administered with phenol red test meal. Data were expressed as mean \pm SEM (Group I - V; n=12 and Group VI-VIII; $n=6$ ). * $p<0.05$ significantly different from normal control group; ${ }^{* *}$ p $<0.01$ significantly different from normal control group.

\section{Discussion}

Dyspepsia in the absence of a clinically identifiable structural lesion is addressed as functional dyspepsia (FD). FD remains one of the most common and costly medical conditions in primary care as well as in gastroenterology practices. The impact of FD remains stressful and leads to an enormous medical expenses. In addition, direct and indirect economic stress due to FD was found to be high and also has considerable impact on productivity. The health related quality of life is impacted significantly in patients with FD when compared to general healthy population $[6,7]$. A disturbance in gastric emptying has been reported to be a major pathophysiological mechanism of FD [1].

Gastric emptying (GE) is the process of transferring the gastric content to the small intestine as the result of motor activity of the stomach, pylorus and duodenum under the control of inhibitory and stimulatory mechanism $[8,1]$. Drugs that enhance GE are effective in the treatment of FD [2]. With the globally increasing popularity of medicinal plants for treating diseases, many herbal extracts/preparations are evaluated for the management of gastrointestinal disorders.

Glycyrrhiza glabra also known as licorice have been traditionally used for many centuries. GutGard is a flavonoid rich, root extract of Glycyrrhiza glabra has been reported to possess a variety of pharmacological activities like anti-dyspeptic, anti-ulcer $\&$ anti-oxidant activities that can be attributed to the benefi- cial effects of GutGard on gastrointestinal system [4,9]. Clinically Gutgard was effective in reducing the symptoms of functional dyspepsia in double blind placebo controlled human clinical trial [4]. With this background on the beneficial effects of Glycyrrhizaglabra on gastrointestinal disorders specifically for anti-dyspeptic activity the present study was conducted to determine the effects ofGutGard on gastric emptying and gastrointestinal transit in normal rats. In addition, the effects of glabridin a phytoactives of GutGard on gastric emptying and gastrointestinal transit were also investigated.

Phenol red recovery model has been widely used method to measure the gastric emptying and gastrointestinal transit in rodents. The model has been used to measure gastric emptying and gastrointestinal transit for both synthetic as well as herbal products [10-15]. Hence, the current study employed phenol red meal model to investigate the possible prokinetic effects of GutGard.

Gastric emptying was measured after administration of a non-absorbable dye (phenol red) as a marker. Gastric emptying was calculated as the percentage of dye remaining in the stomach relative to the total amount of dye recovered in a group of rat that were sacrificed immediately after gavage [16]. The total length of the small intestine and the distance travelled by phenol red meal was measured for obtaining percentage gastrointestinal transit [14]. The percentage gastric emptying and gastrointestinal tran- 
sit were dose dependently enhanced by GutGard and glabridin. Prokinetic activity of Gutgard and glabridin was comparable to domperidone .

In a study conducted by Chen et al, [17] isoliquiritigenin a flavonoid isolated from the roots of Glycyrrhiza glabra has been reported to have antispasmodic and prokinetic effect due to the blockage of the calcium channels. In another study conducted by Khoshnazar et al, [18] the antispasmodic effect of alcoholic extract of licorice rhizome in rat duodenum has been reported to be due to the blockage of the calcium channels or activation of ATP-sensitive potassium channels. In the present study, the effects of GutGard on gastric motility could be due to the presence of glabridin and other flavonoids like isoliquiritigenin.

\section{Conclusion}

The study findings indicate that, GutGard a flavonoid rich root extract of Glycyrrhiza glabra accelerates both gastric emptying and gastrointestinal transit in rats and the effects of GutGard might be contributed by glabridin. GutGard could be a potential lead as prokinetic agent in the management of gastrointestinal disorders which need accelarated gut motility.

\section{Declarations \\ Ethical approval}

All the experimental protocols (Protocol approval number: IAEC/PCL/06/06.15) were approved by the Institutional Animal Ethics Committee (IAEC) of Natural Remedies Private Limited, Bangalore, and conducted according to the Committee for the Purpose of Control and Supervision of Experiments on Animals (CPCSEA) guidelines.

\section{References}

1. Yu YH, Jo Y, Jung JY, Kim BK, Seok JW. Gastric emptying in migraine: a comparison with functional dyspepsia. J Neurogastroenterol Motil. 2012;18(4):412-418. doi: 10.5056/jnm.2012.18.4.412

2. Poudel BK, Yu JY, Kwon YS, Park HG, Son M, Jun JH, et al.The Pharmacological Effects of Benachio- $\mathrm{F}(\AA)$ on Rat Gastrointestinal Functions. Biomol Ther (Seoul). 2015;23(4):350-356. doi: 10.4062/biomolther.2015.035

3. Stanghellini V, De Giorgio R, Barbara G, Cogliandro R, Tosetti C, De Ponti F, et al. Delayed Gastric Emptying in Functional Dyspepsia. Curr Treat Options Gastroenterol. 2004;7(4):259-264.

4. Raveendra KR, Jayachandra, Srinivasa V, Sushma KR, Allan JJ, Goudar KS, et al. An Extract of Glycyrrhiza glabra (GutGard) Alleviates Symptoms of Functional Dyspepsia: A Randomized, Double-Blind, Placebo-Controlled Study. Evid Based Complement Alternat Med. 2012;2012:216970. doi: 10.1155/2012/216970

5. Puram S, Suh HC, Kim SU, Bethapudi B, Joseph JA, Agarwal A, et al. Effect of GutGard in the Management of Helicobacter pylori: A Random- ized Double Blind Placebo Controlled Study. Evid Based Complement Alternat Med. 2013;(2013):1-8.

6. Chang L. Review article: epidemiology and quality of life in functional gastrointestinal disorders. Aliment Pharmacol Ther. 2004;20:31-39.

7. Brook RA, Kleinman NL, Choung RS, Melkonian AK, Smeeding JE, Talley NJ. Functional dyspepsia impacts absenteeism and direct and indirect costs. Clin Gastroenterol Hepatol. 2010;8(6):498-503. doi: 10.1016/j. cgh.2010.03.003

8. NW Weishrodt. Gastric emptying. In: Johnson LR (Editor). Gastrointestinal Physiology. 1997; pp3342.

9. Mukherjee M, Bhaskaran N, Srinath R, Shivaprasad HN, Allan JJ, Shekhar D, et al. Anti-ulcer and antioxidant activity of GutGard. Indian J Exp Biol. 2010;48(3):269-274.

10. Drazigost Pokorn, Cirila HR and Anton Cerar. Gastric Content of the Liquid Test Meals in Rats with Gastrointestinal Neoplasias Induced by $\mathrm{N}$-methyl-N-nitrosoguanidine and 1,2-dimethylhydrazine. International Journal of Cancer Research. 2006;2(1):19-30. DOI: 10.3923/ ijcr.2006.19.30

11. Izbéki F, Wittmann T, Csáti S, Jeszenszky E, Lonovics J. Opposite effects of acute and chronic administration of alcohol on gastric emptying and small bowel transit in rat. Alcohol Alcohol. 2001;36(4):304-308.

12. Tamhane MD, Thorat SP, Rege NN, Dahanukar SA. Effect of oral administration of Terminalia chebula on gastric emptying: an experimental study. J Postgrad Med. 1997;43(1):12-13.

13. Souza MA, Souza MH, Palheta RC Jr, Cruz PR, Medeiros BA, Rola FH, et al. Evaluation of gastrointestinal motility in awake rats: a learning exercise for undergraduate biomedical students. Adv Physiol Educ. 2009;33(4):343-348. doi: 10.1152/advan.90176.2008

14. Srinivasan D, Ramaswamy S, Sengottuvelu S. Prokinetic Effect of Polyherbal Formulation on Gastrointestinal Tract. Phcog Mag. 2009;5(17):37-42.

15. Reynell PC, Spray GH. The simultaneous measurement of absorption and transit in the gastro-intestinal tract of the rat. J Physiol. 1956;131(2):452-462.

16. Firpo MA, Rollins MD, Szabo A, Gull JD, Jackson JD, Shao Y, et al. A conscious mouse model of gastric ileus using clinically relevant endpoints. BMC Gastroenterol. 2005;5:18. DOI: 10.1186/1471-230X-5-18

17. Chen G, Zhu L, Liu Y, Zhou Q Chen H, Yang J. Isoliquiritigenin, a flavonoid from licorice, plays a dual role in regulating gastrointestinal motility in vitro and in vivo. Phytother Res. 2009;23(4):498-506. doi: 10.1002/ptr.2660

18. Seyedeh Mahdieh Khoshnazar, Aminollah Bahaoddini, Hamid Najafipour. Effect of Alcoholic Extract of Licorice (Glycyrrhiza glabra 1.) Rhizome on Isolated Duodenum Motility in Male Rats and its Interference with Cholinergic, Nitrergic, and Adrenergic Systems. Bull Env Pharmacol Life Sci. 2013;2(12):173-177. 